PROCEEDINGS OF THE

AMERICAN MATHEMATICAL SOCIETY

Volume 134, Number 5, Pages 1359-1364

S 0002-9939(05)08080-9

Article electronically published on October 7, 2005

\title{
HANKEL-TYPE OPERATORS ON THE SPACE OF BOUNDED HARMONIC FUNCTIONS
}

\author{
KEIJI IZUCHI AND SHÛICHI OHNO
}

(Communicated by Joseph A. Ball)

Dedicated to Professor Kazuyuki Tsurumi on the occasion of his 70th birthday

\begin{abstract}
We study Hankel-type operators on the space of bounded harmonic functions on the open unit disk. These operators are related to tight uniform algebras, the Dunford-Pettis property, and Bourgain algebras.
\end{abstract}

\section{INTRODUCTION}

Let $X$ be a commutative Banach algebra and let $Y$ be a closed subspace. We define a Hankel-type operator $S_{g}: Y \rightarrow X / Y$ with symbol $g \in X$ by $S_{g} f=g f+Y$ for all $f \in Y$. These operators are a natural generalization of classical Hankel operators on the Hardy space $H^{2}$. The conditions for $S_{g}$ to be compact, weakly compact and completely continuous have been investigated in various function spaces. The problem of whether all $S_{g}$ are weakly compact on a uniform algebra is related to a tight algebra, and the problem of complete continuity appeared in the DunfordPettis property. The latter introduced a notion of Bourgain algebras which have been actively researched in analytic and harmonic function spaces on the open unit disk. Recently, Dudziak, Gamelin, and Gorkin [1 studied Hankel-type operators on analytic function spaces. But the problem of the compactness remains open in the case of the bounded harmonic function space on the open unit disk $\mathbb{D}$. In this paper we study this problem. See [3] and [5] as surveys for convenience.

Let $L^{\infty}(\mathbb{D})$ be the set of all bounded measurable functions on $\mathbb{D}$. Then $L^{\infty}(\mathbb{D})$ is a Banach algebra with the essential supremum norm $\|f\|_{\infty}=$ ess. $\sup \{|f(z)| ; z \in$ $\mathbb{D}\}$. Let $h^{\infty}:=h^{\infty}(\mathbb{D})$ be the set of all bounded harmonic functions on $\mathbb{D}$. It follows that $h^{\infty}$ is a closed subspace of $L^{\infty}(\mathbb{D})$. For $g \in L^{\infty}(\mathbb{D})$, we define the linear operator $S_{g}: h^{\infty} \rightarrow L^{\infty}(\mathbb{D}) / h^{\infty}$ by $S_{g} f=g f+h^{\infty}$ for $f \in h^{\infty}$. Trivially $S_{g}: h^{\infty} \rightarrow L^{\infty}(\mathbb{D}) / h^{\infty}$ is a bounded linear operator. Recall that $S_{g}$ is said to be compact if $S_{g}$ maps every bounded set into a relatively compact one, and that $S_{g}$ is said to be completely continuous if $S_{g}$ maps every weakly convergent sequence into a norm convergent one. In general, every compact operator is completely continuous.

Received by the editors September 15, 2004 and, in revised form, November 29, 2004.

2000 Mathematics Subject Classification. Primary 47B38, 30D55.

Key words and phrases. Hankel-type operators, Banach space of bounded harmonic functions. 
But the converse is not always true. We define the following sets:

$$
\begin{aligned}
& h_{c}^{\infty}=\left\{g \in L^{\infty}(\mathbb{D}): S_{g} \text { is compact }\right\}, \\
& h_{c c}^{\infty}=\left\{g \in L^{\infty}(\mathbb{D}): S_{g} \text { is completely continous }\right\} .
\end{aligned}
$$

Note that $h_{c}^{\infty} \subset h_{c c}^{\infty}$. Although $h^{\infty}$ is not an algebra, these are closed subalgebras of $L^{\infty}(\mathbb{D})$. Especially $h_{c c}^{\infty}$ is called the Bourgain algebra of $h^{\infty}$.

The Bourgain algebra $h_{c c}^{\infty}$ is characterized by Stroethoff, Yale, and the first author $\left[4\right.$. Let $C(\partial \mathbb{D})$ and $L^{\infty}(\partial \mathbb{D})$ be the spaces of continuous and bounded measurable functions on the unit circle $\partial \mathbb{D}$, respectively. For a function $f \in L^{\infty}(\partial \mathbb{D})$, we denote by $\hat{f}$ the Poisson integral of $f$ on $\mathbb{D}$, that is,

$$
\hat{f}(z)=\int_{0}^{2 \pi} f\left(e^{i \theta}\right) P_{z}\left(e^{i \theta}\right) d \theta / 2 \pi,
$$

where $P_{z}$ is the Poisson kernel of $z \in \mathbb{D}$. Then $\hat{f} \in h^{\infty}$. For any nonempty subset $B$ of $L^{\infty}(\partial \mathbb{D})$, we write $\hat{B}=\{\hat{f}: f \in B\}$. It is known that $f$ in $h^{\infty}$ has a boundary function $f^{*}$ on $\partial \mathbb{D}$ and $\widehat{f^{*}}=f$ on $\mathbb{D}$, so that $h^{\infty}=L^{\infty(\partial \mathbb{D})}$. Let $H^{\infty}(\partial \mathbb{D})$ be the space of boundary functions of bounded analytic functions on $\mathbb{D}$. The algebra $Q C$ of bounded quasi-continuous functions on $\partial \mathbb{D}$ is given by

$$
Q C=\left(H^{\infty}(\partial \mathbb{D})+C(\partial \mathbb{D})\right) \cap \overline{\left(H^{\infty}(\partial \mathbb{D})+C(\partial \mathbb{D})\right)} .
$$

Refer to [2] and [6] for more information. For $0<r<1$, we write $\mathbb{D}_{r}=\{|z| \leq r\}$. Put

$$
V=\left\{g \in L^{\infty}(\mathbb{D}):\left\|g \chi_{\mathbb{D} \backslash \mathbb{D}_{r}}\right\|_{\infty} \rightarrow 0 \text { as } r \rightarrow 1^{-}\right\} .
$$

The equality $h_{c c}^{\infty}=\widehat{Q C}+V$ was given as Corollary 3 in [4. In this paper, we show that

Theorem 1.1. $h_{c}^{\infty}=h_{c c}^{\infty}=\widehat{Q C}+V$.

\section{Proof of Theorem 1.1}

To prove Theorem 1.1, we use the following facts which can easily be checked:

(i) $g \in h_{c}^{\infty}$ if and only if $\bar{g} \in h_{c}^{\infty}$,

(ii) $S_{g}$ is compact on $h^{\infty}$ provided that for every sequence $\left\{f_{n}\right\}_{n}$ in $h^{\infty}$ satisfying $\left\|f_{n}\right\|_{\infty} \leq 1$ and $f_{n} \rightarrow 0$ uniformly on compact subsets of $\mathbb{D}$, then there is a subsequence $\left\{f_{n_{j}}\right\}_{j}$ of $\left\{f_{n}\right\}_{n}$ such that $\left\|S_{g} f_{n_{j}}\right\|_{\infty} \rightarrow 0$ as $j \rightarrow \infty$.

Proof of Theorem 1.1. First, we show that

$$
C(\overline{\mathbb{D}})+V \subset h_{c}^{\infty} \subset h_{c c}^{\infty}=\widehat{Q C}+V,
$$

where $C(\overline{\mathbb{D}})$ is the space of continuous functions on the closed unit disk $\overline{\mathbb{D}}$. It is trivial that $V \subset h_{c}^{\infty}$. Since $h_{c}^{\infty}$ is a closed subalgebra of $L^{\infty}(\mathbb{D})$, by (i) it is sufficient to prove that $z \in h_{c}^{\infty}$. Suppose that $f_{n} \in h^{\infty},\left\|f_{n}\right\|_{\infty} \leq 1$ and $f_{n} \rightarrow 0$ uniformly on compact subsets of $\mathbb{D}$. We shall prove that $\left\|S_{z} f_{n_{j}}\right\|_{\infty} \rightarrow 0$ for some subsequence $\left\{f_{n_{j}}\right\}_{j}$ of $\left\{f_{n}\right\}_{n}$.

Write $f_{n}=u_{n}+i v_{n}$, where $u_{n}$ and $v_{n}$ are real-valued harmonic functions on $\mathbb{D}$. Denote by ${ }^{*} u_{n},{ }^{*} v_{n}$ the harmonic conjugate functions of $u_{n}, v_{n}$, with ${ }^{*} u_{n}(0)=$ ${ }^{*} v(0)=0$, respectively. Set

$$
g_{n}=\frac{1}{2}\left(u_{n}+i^{*} u_{n}+i\left(v_{n}+i^{*} v_{n}\right)\right), \quad h_{n}=\frac{1}{2}\left(u_{n}+i^{*} u_{n}-i\left(v_{n}+i^{*} v_{n}\right)\right) .
$$


Then $f_{n}=g_{n}+\overline{h_{n}}$, and it is known that $g_{n}, h_{n} \in H^{2}$, where $H^{2}$ is the Hardy space on $\mathbb{D}$; see Chapter III of Garnett's book [2]. Identifying functions with boundary functions, and denoting by $\|\cdot\|_{2}$ the square integral norm on $\partial \mathbb{D}$, we know that

$$
\left\|^{*} u_{n}\right\|_{2} \leq\left\|u_{n}\right\|_{2} \leq\left\|f_{n}\right\|_{2} \leq\left\|f_{n}\right\|_{\infty} \leq 1, \quad\left\|^{*} v_{n}\right\|_{2} \leq\left\|v_{n}\right\|_{2} \leq 1 .
$$

Hence

$$
\sup _{n}\left\|g_{n}\right\|_{H^{2}} \leq 2, \quad \sup _{n}\left\|h_{n}\right\|_{H^{2}} \leq 2,
$$

where $\|\cdot\|_{H^{2}}$ is the Hardy space norm. By a normal family argument, there exist subsequences (which we do not relabel) of $\left\{g_{n}\right\},\left\{h_{n}\right\}$ such that $g_{n} \rightarrow g, h_{n} \rightarrow h$ uniformly on compact subsets of $\mathbb{D}$ for some analytic functions $g$ and $h$. Since $f_{n} \rightarrow 0$ uniformly on compact subsets of $\mathbb{D}$ and $f_{n}=g_{n}+\overline{h_{n}}, g=-\bar{h}$ on $\mathbb{D}$. Since $g$ and $h$ are analytic, $g=-\bar{h}=\alpha$ for some constant $\alpha$ with $|\alpha| \leq 2$.

Putting

$$
k_{n}=z g_{n}+\overline{\left(\frac{h_{n}-h_{n}(0)}{z}\right)}-\alpha z
$$

then $k_{n}$ is harmonic on $\mathbb{D}$ and

$$
k_{n}=z\left(g_{n}+\overline{h_{n}}\right)-z \overline{h_{n}}+\overline{\left(\frac{h_{n}-h_{n}(0)}{z}\right)}-\alpha z .
$$

Since $f_{n} \in h^{\infty}, z\left(g_{n}+\overline{h_{n}}\right)-\alpha z=z f_{n}-\alpha z \in L^{\infty}(\mathbb{D})$. It is clear that $z \overline{h_{n}}-$ $\overline{\left(\left(h_{n}-h_{n}(0)\right) / z\right)}$ is bounded near the origin of $\mathbb{D}$. By $(2.2)$, we have

$$
\left|h_{n}(z)\right| \leq\left\|h_{n}\right\|_{H^{2}} \frac{1}{\sqrt{1-|z|^{2}}} \leq \frac{2}{\sqrt{1-|z|^{2}}} \quad \text { for } \quad z \in \mathbb{D} .
$$

Since

$$
\left|z \overline{h_{n}}(z)-\overline{\left(\frac{h_{n}(z)-h_{n}(0)}{z}\right)}\right| \leq \frac{\left|h_{n}(z)\right|\left(1-|z|^{2}\right)}{|z|}+\frac{\left|h_{n}(0)\right|}{|z|},
$$

$z \overline{h_{n}}-\overline{\left(\left(h_{n}-h_{n}(0)\right) / z\right)}$ is bounded on $\mathbb{D}$. Thus we get $k_{n} \in h^{\infty}$.

To show that $\left\|z f_{n}-k_{n}\right\|_{\infty} \rightarrow 0$ as $n \rightarrow \infty$, let $\epsilon$ be an arbitrary small positive number and $\delta=\sqrt{1-\epsilon^{2}}$. By (2.3), we have

$$
\left\|z f_{n}-k_{n}\right\|_{\infty}=\left\|z \overline{\left(h_{n}+\bar{\alpha}\right)}-\overline{\left(\frac{h_{n}+\bar{\alpha}-\left(h_{n}(0)+\bar{\alpha}\right)}{z}\right)}\right\|_{\infty} .
$$

Since $h_{n} \rightarrow-\bar{\alpha}$ uniformly on $\mathbb{D}_{\delta}$,

$$
\sup _{z \in \mathbb{D}_{\delta}}\left|\overline{z\left(h_{n}(z)+\bar{\alpha}\right)}-\overline{\left(\frac{h_{n}(z)+\bar{\alpha}-\left(h_{n}(0)+\bar{\alpha}\right)}{z}\right)}\right| \rightarrow 0
$$

as $n \rightarrow \infty$. For $|z|>\delta$, we have

$$
\begin{aligned}
& \left|z \overline{\left(h_{n}(z)+\bar{\alpha}\right)}-\overline{\left(\frac{h_{n}(z)+\bar{\alpha}-\left(h_{n}(0)+\bar{\alpha}\right)}{z}\right)}\right| \\
& =\left|\frac{1-|z|^{2}}{\bar{z}} \overline{\left(h_{n}(z)+\bar{\alpha}\right)}-\frac{\overline{h_{n}(0)+\bar{\alpha}}}{\bar{z}}\right| \\
& \leq \frac{\epsilon \mid h_{n}+\bar{\alpha} \|_{H^{2}}}{\delta}+\frac{\left|h_{n}(0)+\bar{\alpha}\right|}{\delta} \quad \text { by }(2.4) \\
& <\frac{4 \epsilon}{\delta}+\frac{\left|h_{n}(0)+\bar{\alpha}\right|}{\delta} \quad \text { by }(2.2) \text { and }|\alpha| \leq 2 .
\end{aligned}
$$


Combining with (2.5),

$$
\limsup _{n \rightarrow \infty}\left\|z f_{n}-k_{n}\right\|_{\infty} \leq \frac{4 \epsilon}{\delta}=\frac{4 \epsilon}{\sqrt{1-\epsilon^{2}}} .
$$

Since $\epsilon$ is arbitrary,

$$
\lim _{n \rightarrow \infty}\left\|S_{z} f_{n}\right\|_{\infty} \leq \lim _{n \rightarrow \infty}\left\|z f_{n}-k_{n}\right\|_{\infty}=0 .
$$

Thus by (ii), $z \in h_{c}^{\infty}$, and it holds that

$$
C(\overline{\mathbb{D}})+V \subset h_{c}^{\infty} \subset h_{c c}^{\infty}=\widehat{Q C}+V .
$$

Next we show that for any $g \in Q C, \hat{g} \in h_{c}^{\infty}$, that is, $S_{\hat{g}}$ is compact on $h^{\infty}$. To show this, note the equality $h^{\infty}=L^{\infty}(\partial \mathbb{D})$. Let $\left\{f_{n}\right\}_{n}$ be a sequence in $h^{\infty}$ satisfying that $\left\|f_{n}\right\|_{\infty} \leq 1$ and $\hat{f}_{n} \rightarrow 0$ uniformly on compact subsets of $\mathbb{D}$. We have $\left\|S_{\hat{g}} \hat{f}_{n}+h^{\infty}\right\| \leq\left\|\hat{g} \hat{f}_{n}-\widehat{g f_{n}}\right\|_{\infty}$ and

$$
\left|\hat{g}(z) \hat{f}_{n}(z)-\widehat{g f_{n}}(z)\right| \leq \int_{0}^{2 \pi}\left|\hat{g}(z)-g\left(e^{i \theta}\right)\right| P_{z}(\theta) d \theta / 2 \pi .
$$

Since $g \in Q C$, we have

$$
\sup _{r<|z|<1} \sup _{n}\left|\hat{g}(z) \hat{f}_{n}(z)-\widehat{g f_{n}}(z)\right| \rightarrow 0 \quad \text { as } r \rightarrow 1 ;
$$

see [2, 4, 6]. Then for any $\varepsilon>0$, there exists $R, 0<R<1$, such that

$$
\sup _{R<|z|<1} \sup _{n}\left|\hat{g}(z) \hat{f}_{n}(z)-\widehat{g f_{n}}(z)\right|<\varepsilon .
$$

On the other hand, by the assumption, $\sup _{z \in \mathbb{D}_{R}}\left|\hat{g}(z) \hat{f}_{n}(z)\right| \rightarrow 0$ as $n \rightarrow \infty$. Now we define $g_{t}\left(e^{i \theta}\right)=\hat{g}\left(t e^{i \theta}\right)$ for $0<t<1$. Then $g_{t} \in C(\partial \mathbb{D}), \hat{g}_{t}(z)=\hat{g}(t z)$ for $z \in \mathbb{D}$, and

$$
\begin{aligned}
\sup _{z \in \mathbb{D}_{R}}\left|\widehat{g f_{n}}(z)-\widehat{g_{t} f_{n}}(z)\right| & \leq \sup _{z \in \mathbb{D}_{R}} \int_{0}^{2 \pi}\left|g\left(e^{i \theta}\right)-g_{t}\left(e^{i \theta}\right)\right|\left|f_{n}\left(e^{i \theta}\right)\right| P_{z}\left(e^{i \theta}\right) d \theta / 2 \pi \\
& \leq \frac{1+R}{1-R} \int_{0}^{2 \pi}\left|g\left(e^{i \theta}\right)-g_{t}\left(e^{i \theta}\right)\right| d \theta / 2 \pi .
\end{aligned}
$$

By the Lebesgue dominated convergence theorem,

$$
\lim _{t \rightarrow 1} \sup _{n} \sup _{z \in \mathbb{D}_{R}}\left|\widehat{g f_{n}}(z)-\widehat{g_{t} f_{n}}(z)\right|=0 .
$$

Take a positive number $t_{0}$ such that $t_{0}<1$ and

$$
\sup _{n} \sup _{z \in \mathbb{D}_{R}}\left|\widehat{g f_{n}}(z)-\widehat{g_{t_{0}} f_{n}}(z)\right|<\varepsilon .
$$

By (2.1), $\hat{g}_{t_{0}} \in C(\overline{\mathbb{D}}) \subset h_{c}^{\infty}$, so that by considering a subsequence we may assume that $\left\|\hat{g}_{t_{0}} \hat{f}_{n}+h^{\infty}\right\|_{\infty} \rightarrow 0$ as $n \rightarrow \infty$. Then

$$
\left\|\hat{g}_{t_{0}} \hat{f_{n}}-\widehat{g_{t_{0}} f_{n}}+h^{\infty}\right\|_{\infty} \rightarrow 0 \quad \text { as } n \rightarrow \infty .
$$

We want to prove that

$$
\sup _{z \in \mathbb{D}_{R}}\left|\widehat{g_{t_{0}} f_{n}}(z)\right| \rightarrow 0 \quad \text { as } n \rightarrow \infty .
$$


Let $\left\{h_{n}\right\}_{n}$ be a sequence in $h^{\infty}$ satisfying

$$
\left\|\hat{g}_{t_{0}} \hat{f}_{n}-\widehat{g_{t_{0}} f_{n}}+h_{n}\right\|_{\infty}=\left\|\hat{g}_{t_{0}} \hat{f}_{n}-\widehat{g_{t_{0}} f_{n}}+h^{\infty}\right\|_{\infty} .
$$

Since $g_{t} \in C(\partial \mathbb{D}) \subset Q C$, by $(2.6)$

$$
\left\|\hat{g}_{t_{0}} \hat{f}_{n}-\widehat{g_{t_{0}} f_{n}}+h_{n}\right\|_{\infty} \geq \limsup _{|z| \rightarrow 1}\left|h_{n}(z)\right|=\left\|h_{n}\right\|_{\infty} .
$$

By $(2.9)\left\|h_{n}\right\|_{\infty} \rightarrow 0$, and by $(2.11)$

$$
\left\|\hat{g}_{t_{0}} \hat{f}_{n}-\widehat{g_{t_{0}} f_{n}}\right\|_{\infty} \rightarrow 0 \quad \text { as } n \rightarrow \infty .
$$

We have

$$
\left\|\hat{g}_{t_{0}} \hat{f}_{n}-\widehat{g_{t_{0}} f_{n}}\right\|_{\infty} \geq \sup _{z \in \mathbb{D}_{R}}\left\|\widehat{g_{t_{0}} f_{n}}(z)|-| \hat{g}_{t_{0}}(z) \hat{f}_{n}(z)\right\| .
$$

By the assumption, $\sup _{z \in \mathbb{D}_{R}}\left|\hat{g}_{t_{0}}(z) \hat{f}_{n}(z)\right| \rightarrow 0$ as $n \rightarrow \infty$. Then by (2.12), we get (2.10).

Combining (2.8) and (2.10), we have

$$
\limsup _{n \rightarrow \infty} \sup _{z \in \mathbb{D}_{R}}\left|\widehat{g f_{n}}(z)\right| \leq \epsilon
$$

This shows that $\sup _{z \in \mathbb{D}_{R}}\left|\widehat{g f_{n}}(z)\right| \rightarrow 0$ as $n \rightarrow \infty$. Since $\sup _{z \in \mathbb{D}_{R}}\left|\hat{g}(z) \hat{f}_{n}(z)\right| \rightarrow 0$ by our assumption, we get

$$
\sup _{z \in \mathbb{D}_{R}}\left|\hat{g}(z) \hat{f}_{n}(z)-\widehat{g f_{n}}(z)\right| \rightarrow 0 \quad \text { as } n \rightarrow \infty .
$$

Combining with (2.7), we have

$$
\limsup _{n \rightarrow \infty}\left\|\hat{g} \hat{f}_{n}-\widehat{g f_{n}}\right\|_{\infty} \leq \epsilon .
$$

Consequently we obtain

$$
\left\|S_{\hat{g}} \hat{f}_{n}+h^{\infty}\right\| \leq\left\|\hat{g} \hat{f}_{n}-\widehat{g f_{n}}\right\|_{\infty} \rightarrow 0
$$

that is, $S_{\hat{g}}$ is compact and so $\widehat{Q C} \subset h_{c}^{\infty}$. We complete the proof.

\section{ACKNOWLEDGMENTS}

The first author was partially supported by Grant-in-Aid for Scientific Research (No. 16340037), Ministry of Education, Science and Culture. The second author was partially supported by Grant-in-Aid for Scientific Research (No. 15540181), Ministry of Education, Science and Culture. The second author also thanks Professor K. Izuchi for his hospitality while he visited Department of Mathematics, Niigata University, where the work for part of this paper was done.

\section{REFERENCES}

1. J. Dudziak, T. W. Gamelin and P. Gorkin, Hankel operators on bounded analytic functions, Trans. Amer. Math. Soc. 352 (2000), 363-377. MR1473437 (2000c:47049)

2. J. B. Garnett, Bounded Analytic Functions, Academic Press, New York, 1981. MR0628971 (83g:30037)

3. P. Gorkin, Hankel-type operators, Bourgain algebras, and uniform algebras, Holomorphic Spaces (Berkeley, CA, 1995), 121-134, Math. Sci. Res. Inst. Publ. 33, Cambridge Univ. Press, Cambridge, 1998. MR 1630647 (99g:47052)

4. K. Izuchi, K. Stroethoff and K. Yale, Bourgain algebras of spaces of harmonic functions, Michigan Math. J. 41 (1994), 309-321. MR1278437(95d:46051) 
5. S. Saccone, Tight uniform algebras, Holomorphic Spaces (Berkeley, CA, 1995), 135-154, Math. Sci. Res. Inst. Publ. 33, Cambridge Univ. Press, Cambridge, 1998. MR 1630648 (2000a:46085)

6. D. Sarason, Functions of vanishing mean oscillation, Trans. Amer. Math. Soc. 207 (1975), 391-405. MR0377518 (51:13690)

Department of Mathematics, Nitgata University, Niigata, 950-2181, Japan

E-mail address: izuchi@math.sc.niigata-u.ac.jp

Nippon Institute of Technology, Miyashiro, Minami-Saitama 345-8501, Japan

E-mail address: ohno@nit.ac.jp 